\title{
Plasma Transfusion Practice in Adult Surgical Patients: Systematic Review of the Literature
}

\author{
Elisabeth Hannah Adam ${ }^{a}$ Dania Fischer ${ }^{b}$ \\ ${ }^{a}$ Department of Anesthesiology, Intensive Care Medicine and Pain Therapy, University Hospital Frankfurt, Goethe

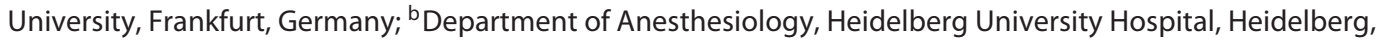 \\ Germany
}

\section{Keywords}

Fresh frozen plasma - Plasma transfusion - Plasma usage . Coagulopathy management

\begin{abstract}
Background: Plasma transfusions are most commonly used therapeutically for bleeding or prophylactically in nonbleeding patients prior to invasive procedures or surgery. Although plasma transfusions generally seem to decline, plasma usage for indications that lack evidence of efficacy prevail. Summary: There is wide international, interinstitutional, and interindividual variance regarding the compliance with guidelines based on published references, supported by appropriate testing. There is furthermore a profound lack of evidence from randomized controlled trials comparing the effect of plasma transfusion with that of other therapeutic interventions for most indications, including massive bleeding. The expected benefit of a plasma transfusion needs to be balanced carefully against the associated risk of adverse events. In light of the heterogeneous nature of bleeding conditions and their rapid evolvement over time, fibrinogen and factor concentrate therapy, directed at specific phases of coagulation identified by alternative laboratory assays, may offer advantages over conventional blood product ratio-driven resuscitation. However, their outcome benefit has not been demonstrated in well-powered prospective trials. This systematic review will detail the current evidence base for plasma transfusion in adult surgical patients.
\end{abstract}

(c) 2020 S. Karger AG, Basel

\section{Introduction}

The indications for plasma transfusion are very limited. In the perioperative setting, plasma transfusion is commonly used to treat and prevent bleeding. However, there is ample evidence for both inadequate dosing and unnecessary plasma usage for indications not substantiated by robust trial data.

In this overview, we provide an update of the recent literature to identify best-care practices and evaluate measures to curtail non-evidence-based plasma transfusion with specific emphasis on surgical patients.

\section{Plasma Preparations for Transfusion}

The preparation of human plasma products follows strict guidelines from the point of donor selection to product processing. Fresh frozen plasma (FFP) is produced from whole blood donations which undergo centrifugation, or by apheresis [1]. Leukocyte depletion is either achieved by filtration during whole blood processing or integrated in the apheresis process. An anticoagulant solution is always added during the collection of FFP. FFP is quick-frozen to a temperature below $-30^{\circ} \mathrm{C}$ as soon as possible after being obtained but latest within 8 h. FP24 is supernatant plasma expressed and frozen 8-24 h after phlebotomy. Quarantine storage, followed by a second donor screening for infection markers prior to releasing the plasma for treatment, is used to minimize the risk of transfusion-transmitted diseases. Lyophilized plasma 
(LP) is also a single-donor plasma that is lyophilized after quarantine storage and cell filtration and is only solubilized immediately before use. In contrast to FFP, LP can be stored at ambient temperatures, can be reconstituted and administered quickly, resulting in a rapid time-totransfusion [2]. Liquid plasma, on the other hand, is not frozen, but stored at $1-6^{\circ} \mathrm{C}$, limiting its shelf life to 26 days.

In addition to many other substances, plasma contains components for blood clotting, i.e. both the coagulation factors and their inhibitors. During product processing, maintenance of the activity of labile coagulation factors is highly important, factor VIII (FVIII) being the most labile coagulation factor. As a quality control standard, FFPs must contain at least $0.7 \mathrm{IU} / \mathrm{mL}$ of FVIII in at least $75 \%$ of units immediately after thawing. FFP units furthermore contain normal levels of albumin and immunoglobulins as well as all clotting factors and inhibitors, with widely diverging values corresponding to variability between individual donors. Generally, there is an overall reduction in the activity of coagulation factors and their inhibitors associated with processing: mean FII activity immediately after thawing has been reported with $73 \%$ [3] and protein S with 58\% [4]. Factor V (-14\%), X $(-11 \%)$, and XIII $(-20 \%)$ are also significantly decreased after thawing of FFP [5]. Levels of the acute-phase proteins fibrinogen and factor VIII in the plasma show particularly wide variation. FFP obtained by apheresis contains substantially greater activities of factors V, VIII, IX and XI than FFP obtained from whole blood [6].

Lyophilization has no effect on fibrinogen, protein S, or antithrombin activities; however, $10-25 \%$ losses in activity were noted for factors V, VIII, XI, vWF, and plasminogen, as well as a $10 \%$ prolongation in activated partial thromboplastin time (aPTT) [7]. In a randomized open-label trial comparing pathogen-reduced LP with FFP in the initial management of trauma-induced coagulopathy showed that LP achieved a more rapid, pronounced and extended increase in fibrinogen concentrations and a more rapid coagulopathy improvement than FFP [8].

Next to desirable components, plasma may also contain drug residues as shown in a study examining 100 single-donor plasma samples [9]: 12\% of samples yielded detectable amounts of drug residues at concentrations estimated to levels common to individuals under therapeutic treatment. Five were found to contain diuretics ( 4 hydrochlorothiazide and 1 torasemide), 5 contained beta-receptor blocking agents (4 bisoprolol and 1 metoprolol), 1 was found with residues of pseudoephedrine (stimulant) and 1 with drospirenone (contraceptive); 99\% contained caffeine and $6 \%$ nicotine findings.

To minimize the risk of transfusion-transmitted diseases, virus inactivation of plasma is available, production pro- cesses use either solvent-detergent (SD), methylene blue, amotosalen, and riboflavin as additives [10]. The availability of different plasma products varies between countries. All systems result in good reduction of enveloped viruses (generally $>4 \log$ ), but activity against non-enveloped viruses (hepatitis A virus, parvovirus B19, and hepatitis E virus) is more variable. All methods result in a reduction in protein values with the percent retention of FVIII activity in the range of $67-78 \%$ and fibrinogen of $65-84 \%$. Alterations in fibrinogen structure have been reported with methylene blue [11]. SD plasma has a low batch-to-batch variation as it is pooled from $500-1,600$ donors. It contains markedly lower protein $S$ and plasmin inhibitor activity than standard FFP and no $\alpha_{1}$-antitrypsin [12]. One study by Haubelt et al. [13] compared the effect of SD plasma and FFP on hemostasis and fibrinolysis in complex coagulopathy after open-heart surgery. With the exception of protein $S$ and plasmin inhibitor, SD plasma and FFP improved hemostasis and fibrinolysis to a similar degree.

\section{Plasma-Derived Medicines}

Although the various coagulation factors are present at physiological concentrations in FFP derived from healthy blood donors, some virally inactivated plasmaderived coagulation factor concentrates have been available for many years. These latter products include single coagulation factor concentrates (such as fibrinogen, factor VIII concentrates for the treatment of hemophilia A, and factor IX concentrate for the treatment of hemophilia B) or the so-called prothrombin complex concentrates (PCC), which are intermediate purity pooled plasma products containing a mixture of vitamin $\mathrm{K}$-dependent proteins [14]. Fibrinogen concentrate is a virally inactivated product made from pooled human plasma. Although the precise amount of fibrinogen can vary among formulations from different sources, the fibrinogen content of fibrinogen concentrate is $15-20 \mathrm{mg} / \mathrm{mL}$. PCC is a lyophilized, nonactivated plasma protein concentrate prepared from pooled human plasma. PCC contains FII, FIX, and FX (3F-PCC) and, depending on the formulation, FVII (4F-PCC). It provides a concentration of clotting factors approximately $25 \times$ that of plasma. Availability, composition, and costs of plasma derived medicines differ between healthcare systems.

\section{Current Recommendations from Guidelines for the Use of FFP}

Plasma transfusion guidelines come from multiple sources; all of them criticize the low level of clinical evidence, which complicates the findings of consensus. Dif- 
ferences in recommendations may further be explained by the availability of resources and affordability of plasma and plasma-derived medicines in the national markets.

Joint recommendations of guidelines we chose as examples are summarized below, specific recommendations elaborated in more detail. Some guidelines use the GRADE criteria, details on which can be found at http:// www.gradeworkinggroup.org.

\section{Australia}

The world-first Australian Patient Blood Management (PBM) program is firmly anchored in guidelines by the National Blood Authority of Australia [15]. These state, in unison with all other guidelines, that FFPs are recommended in patients with critical bleeding requiring massive transfusion and for warfarin reversal in situations where PCC is not available. The prophylactic use of FFP in cardiac surgery is not recommended (GRADE B). The routine use of FFP in medical patients with coagulopathy (including those with hepatic impairment) and critically ill patients with coagulopathy is not advised. The National Blood Authority of Australia underlines that laboratory coagulation analyses correlate poorly with bleeding risk in hepatic impairment. Furthermore, patients with an international normalized ratio (INR) $\leq 2$ "may not benefit from the administration of FFP and can generally undergo invasive procedures within the ICU without any serious bleeding; higher INRs may be tolerated in certain clinical situations."

\section{USA}

The current AABB (formerly American Association of Blood Banks) guideline similarly found that there were inadequate clinical data to recommend plasma transfusion with the exception of plasma use in the setting of massive transfusion and in patients with warfarin-related intracranial hemorrhage, albeit with a low-quality of evidence related to the latter recommendation [16]. The AABB clearly suggests against transfusing plasma in other groups of patients such as "acute pancreatitis, organophosphate poisoning, coagulopathy associated with acetaminophen overdose, intracranial hemorrhage after severe closed head injury in patients without coagulopathy, nonsurgical noncardiac patients in the intensive care unit; quality of evidence = very low)." In these cases, the guideline warns, the adverse effects of plasma transfusion may outweigh any potential benefits.

\section{$U K$}

The British Society of Haematology Guidelines focus on clinical scenarios in the absence of major bleeding [17]. These also underline that abnormal standard coagulation tests (prothrombin time [PT]/aPTT) are poor predictors of bleeding risks in non-bleeding patients prior to an invasive procedure (2C). Instead, they recommend that a detailed personal and family bleeding history, drug history, and the bleeding risk associated with the planned procedure must be assessed as a matter of routine for all patients undergoing a planned procedure (1B). Standard coagulation tests should be considered in patients undergoing procedures with a moderate or high bleeding risk, any patients on anticoagulants, or those who have a personal/family bleeding history (1B). Patients with a positive personal/family bleeding history should be discussed with hematology as standard clotting test results may be normal in the presence of a significant bleeding tendency (1B). The impact of commonly used doses of FFP to correct clotting results, or to reduce the bleeding risk, is very limited particularly when the PT ratio or INR are between 1.5 and 1.9 (2C). Plasma should not be used for volume replacement $(2 \mathrm{C})$.

There is no evidence to support prophylactic use of FFP in non-bleeding patients with pre-procedural abnormal standard coagulation tests (2C). Invasive procedures may be replaced by alternative procedures with a lower bleeding risk (e.g., transjugular instead of percutaneous liver biopsy) (2C). Vitamin K should be administered in patients with prolonged PT that is likely to be due to acquired vitamin K deficiency (1B).

Another Guideline by the British Society for Haematology recommends the use of viscoelastic hemostatic assays in the management of major bleeding as standard laboratory tests are perceived too slow for a rapidly evolving situation [18].

\section{Germany}

The German guidelines stress that even high doses of plasma only result in a moderate increase in clotting factor and inhibitor activities in the recipient [19]. Therefore, a sufficiently high FFP dose and transfusion speed is required for any effective plasma therapy (a minimum of $15 \mathrm{~mL} / \mathrm{kg}$ body weight, infusion rate $30-50 \mathrm{~mL} / \mathrm{min}$ ). In adults, any dose below $600 \mathrm{~mL}$ (2-3 units) is inadequate [20]. In patients with impaired renal function, severe liver damage, or cardiopulmonary insufficiency, the plasma dose is limited due to the risk of hypervolemia. With regard to dosing, SD plasma contains lower concentrations of clotting factors, so it is recommended to increase the dose by approx. $10 \%$ in comparison to FFP [12].

The German guidelines likewise state that in emergency situations, oral anticoagulants are to be primarily reversed with PCC as it is more rapid and more effective. However, PCC concentrates are no replacement for plasma when treating complex coagulopathies since they do not contain fibrinogen, factor V, factor VIII, vWf, factor $\mathrm{XI}$, and factor XIII. Plasma shall not be transfused as prophylaxis in patients with liver dysfunction and coagulation disorders in the context of fine-needle liver biopsy, 
after paracentesis, thoracentesis, or central venous cannulation $(1 \mathrm{C}+)$. In patients undergoing liver transplantation with $\mathrm{PT} \geq 50 \%$, plasma should not be administered perioperatively as prophylaxis $(2 \mathrm{C}+)$. In patients with liver dysfunction and coagulation disorders, plasma could be transfused when PT is below $50 \%$ and major bleeding occurs at a dose of $20 \mathrm{~mL} / \mathrm{kg}$ body weight. The objective of treatment is to arrest bleeding and to increase PT to at least $50 \%(2 \mathrm{C})$.

In the absence of coagulation deficiencies and active bleeding, FFP should not be used as a volume expander or for the substitution of immunoglobulins (both $1 \mathrm{C}+$ ). Further general recommendations are that transfusion efficacy following plasma transfusion should be controlled by laboratory analyses and suitable transfusion intervals be defined.

\section{Further International/PBM Guidelines}

European Society of Anaesthesiology (ESA) Bleeding Management Guidelines [21]

The ESA recommends pathogen inactivation for FFP and platelets (PLT) (1C). Furthermore, early and targeted treatment of coagulation factor deficiencies is recommended. Depending on the clinical situation, type of bleeding, type of deficiency and resources provided, coagulation factor concentrates, cryoprecipitate or high volumes of plasma shall be used for this (1B). In the treatment of acquired coagulation factor deficiency, the consideration of a ratio-driven protocol (the ratio of red blood cell [RBC] to FFP and to PLT) early in uncontrolled massive bleeding outside the trauma setting is suggested, followed by a goal-directed approach as soon as possible (2C). Coagulation factor concentrates are recommended for the primary treatment of acquired coagulation factor deficiency (2C). The ESA guidelines recommend against indiscriminate use of plasma transfusion in perioperative bleeding management (1C).

In vitamin $\mathrm{K}$ antagonist (VKA)-treated patients undergoing an emergency procedure, the guidelines recommend that INR must be measured on the patient's admission to the hospital, with the administration of $4 \mathrm{~F}-\mathrm{PCC}$ to reverse VKA anticoagulant effects (e.g., at an initial dose of $25 \mathrm{IU}$ factor IX/kg at an INR of 4) rather than the transfusion of plasma (1B). If PCC is not available, then in bleeding patients where VKA-induced coagulopathy is considered a contributing factor, the transfusion of plas$\mathrm{ma}(15-20 \mathrm{~mL} / \mathrm{kg}$ plus $5-10 \mathrm{mg}$ IV vitamin $\mathrm{K})$ is recommended (1C). The ESA guidelines furthermore recommend the application of intervention algorithms incorporating pre-defined triggers and targets based on viscoelastic hemostatic assay coagulation monitoring to guide individualized hemostatic intervention in the case of perioperative bleeding (1C).
Guidelines Endorsed by the Royal College of

Anaesthetists and the Network for Advancement of

Transfusion Alternatives (NATA) Established by

the Association of Anaesthetists of Great Britain and Ireland (AAGBI) [22]

Viscoelastic testing is recommended to guide transfusion. Indications for FFP use include the following: replacement of coagulation factors during major hemorrhage, particularly trauma and obstetrics; acute disseminated intravascular coagulation with bleeding; in patients who are actively bleeding and whose INR is $>1.5$ (or POC equivalent); immediate reversal of warfarin-induced hemorrhage when PCC is not available (PCC is the first choice); replacement of coagulation factors when specific factors are not available (uncommon). The guidelines stress that there is a very limited role for FFP in the management of (mild-moderate) coagulation abnormalities frequently seen in many non-bleeding critically ill patients before invasive procedures. FFP is not recommended for routine use in patients with cirrhosis/liver disease unless significant coagulopathy is identified, as again current understanding indicates that isolated abnormalities of the PT or aPTT do not reflect a "balanced hemostasis." FFP should not be used simply as routine circulatory volume replacement.

The International Society on Thrombosis and Haemostasis (ISTH): Postpartum Hemorrhage

Guidelines [23]

The guidelines emphasize that coagulopathies associated with postpartum hemorrhage differ from those associated with trauma-induced massive hemorrhage. It is recommended that hemostasis may be assessed by: (i) clinical observation, (ii) laboratory-based PT/aPTT, Clauss fibrinogen and platelet count, and (iii) point-ofcare testing, stressing that no high-level data suggest that any strategy is better and all three may be used simultaneously.

\section{EACTS/EACTA PBM Guidelines [24]}

FFP might be used to reverse the action of oral anticoagulation (2B) or in the case of persistent perioperative bleeding, but the guidelines stress that there is no evidence that prophylactic or therapeutic FFP transfusions reduce blood loss after cardiac surgery. PBM generally advocates an individualized approach, tailored to each patient's needs.

\section{Society of Cardiovascular Anesthesiologists (SCA)}

Guidelines [25]

The guidelines focus on cardiac surgery and recommend that FFP transfusion is indicated if excessive bleeding with coagulation factor deficiency and/or if ROTEM/ TEG show signs of factor deficiency. FFP transfusion may 
furthermore be considered if part of massive transfusion algorithm. Is not indicated for urgent warfarin reversal when PCC is available or no active bleeding. Is not indicated for volume replacement. Prophylactic administration of FFP during cardiac surgery, without evidence of coagulation factor deficiency, is not effective in reducing post-CPB (cardiopulmonary bypass) bleeding and is not recommended.

The guidelines recommend the application of transfusion algorithms incorporating predefined intervention triggers based on point-of-care coagulation monitoring assays to guide hemostatic intervention. It stresses that the implementation of transfusion and coagulation management algorithms (based on ROTEM/TEG) and goaldirected therapy with coagulation factor concentrates may reduce transfusion-associated adverse events.

European Society of Intensive Care Medicine (ESICM) Guidelines [26].

The ESICM guidelines address transfusion strategies in non-bleeding critically ill patients. As regards plasma transfusion in this patient subgroup, the guidelines suggest not giving prophylactic plasma transfusion in patients with coagulopathy (conditional recommendation, very low certainty) and also suggest against the use of prophylactic plasma transfusion prior to invasive bedside procedures in non-bleeding critically ill patients (conditional recommendation, very low certainty).

\section{RCTs Assessing the Efficacy and Safety of FFP}

Plasma is transfused extensively in a variety of settings from massive transfusion to VKA reversal. Despite the widespread use of plasma as a hemostatic agent in bleeding patients, its effect in comparison with other available choices of hemostatic therapies is unclear, and randomized controlled trials that assessed the efficacy and/or safety of therapeutic plasma as an intervention to treat bleeding patients compared with other interventions or placebo are scarce [27]. In the following section, relevant publications are summarized according to indication.

\section{Massive Bleeding}

Massive hemorrhage occurs in many clinical settings including obstetrics, gastroenterology, trauma, and the operating room. Massive transfusion is an important lifesaving intervention for patients with massive acute blood loss.

The discussion concerning the plasma volumes to be transfused and the ratio of RBC to FFP and to PLT has been ongoing for years. Especially in trauma patients, an aggressive therapy of coagulopathy, seems to be advantageous to improve outcome [28-32]. Although the trans- fusion of plasma seems intuitively consistent in the context of massive bleeding, in a prospective study by Scalea et al. [33] a beneficial effect has not been demonstrated for trauma patients transfused aggressively with FFPs. In this study, 806 consecutive trauma patients admitted to the intensive care unit over 2 years were stratified by the RBC:FFP transfusion ratio over the first $24 \mathrm{~h}$. There was no significant difference in outcome when comparing patients who had a 1:1 RBC:FFP ratio with those who did not receive any FFP. The PROPPR Trial in 680 patients with severe trauma and major bleeding compared a 1:1:1 (FFP:PLT:RBC) with a 1:1:2 ratio and found no significant differences in mortality at $24 \mathrm{~h}$ or at 30 days. However, more patients in the 1:1:1 group achieved hemostasis ( 86 vs. $78 \%$, respectively; $p=0.006$ ), and fewer experienced death due to exsanguination by $24 \mathrm{~h}$ ( 9.2 vs. $14.6 \%$ in the 1:1:2 group; difference, $-5.4 \%$ [ $95 \% \mathrm{CI}-10.4 \%$ to $-0.5 \%] ; p=0.03)$ [34].

Two clinical trials on pre-hospital plasma in trauma showed conflicting results. The multicenter, cluster-randomized PAMPer trial assessed the efficacy and safety of two units of pre-hospital plasma versus standard care without plasma in 501 trauma patients at risk for hemorrhagic shock during air medical transport to a designated US trauma center [35]. The PAMPer trial demonstrated a significantly lower mortality at 30 days in the plasma group than in the standard-care group (23.2 vs. $33.0 \%$; $95 \%$ CI -18.6 to $-1.0 \%$; $p=0.03$ ). However, the randomized, placebo-controlled COMBAT trial compared the same plasma volume versus isotonic saline in 144 hemorrhagic shocked trauma patients and showed the opposite effect in mortality at 28 days ( $15 \%$ in the plasma group vs. $10 \%$ in the control group, $p=0.37$ ) [36]. There are a number of limitations related to both studies. Firstly, a single hypotensive episode irrespective of possible other nonbleeding causes was used as inclusion criterion for both studies. Secondly, a large proportion of patients did not receive massive transfusion and most arrived at the hospital with the absence of clinical/laboratory signs of relevant coagulopathy. Of those who were massively transfused, no difference in mortality was reported independent of pre-hospital plasma administration. Furthermore, in both trials, no relevant improvements in standard/viscoelastic coagulation assays were reported after pre-hospital plasma. In PAMPer, $42 \%$ of the standard care patients had received pre-hospital RBCs versus $26 \%$ in the FFP group and almost twice as much crystalloids prior to hospital admission. The PAMPer study does not provide a statement on the transfusion trigger for RBCs or the percentage of patients receiving tranexamic acid (TXA), while for COMBAT $10 \%$ of patients had received TXA. Additionally, there was a high mortality difference within both control arms, which is presumably due to differences in patient cohort and patterns of care, but leaves us in 
doubt whether the blind pre-hospital administration of plasma to potentially non-coagulopathic patients provides any survival benefit [37]. Further studies in prehospital emergency care are under way, testing the capability of rapidly available French LP, which is storable at room temperature and compatible with all blood types, to reduce the severity of trauma-induced coagulopathy [38].

Two meta-analyses, published in 2004 [39] and updated by the same authors in 2012 [40], did not identify a benefit in favor of the transfusion of FFP in massive bleeding and found only poorly supported evidence that the therapeutic use of plasma is effective. Also, Murad et al. [41] demonstrated in their meta-analysis from 2010 that only limited evidence for FFP transfusion in the setting of massive traumatic bleeding exists for both survival and multiorgan failure. A further meta-analysis in 2017 by Levy et al. [27] could not identify a single randomized controlled trial that investigated the safety and effectiveness of plasma in coagulopathic bleeding.

In 2018, McQuilten et al. [42] reviewed in their metaanalysis the effect of dose, timing and ratio to RBCs in the therapy with blood components in patients with critical bleeding in the setting of massive transfusion. Similar to a recent meta-analysis from 2019 by de Luz et al. [43] the authors could not identify any difference in mortality and morbidity at a transfusion ratio of 1:1:1 (FFP:PLT:RBC) compared to a transfusion ratio of 1:1:2 (FFP:PLT:RBC). Fixed higher ratios of FFP and PLT to RBC are associated with a higher transfusion of FFP and PLT without demonstrating clinical benefit compared to standard care or a 1:1:2 ratio. Due to this limited evidence for RCTs, a 1:1: 1 ratio versus a 1:1:2 ratio or standard care for adult patients with critical bleeding cannot currently be recommended. Even the Pragmatic, Randomized Optimal Platelet and Plasma Ratios (PROPPR) study as one of the largest randomized trials reported no mortality benefit in patients with severe trauma randomized to higher versus lower ratio of plasma and PLT to RBC units, although more patients in the former arm achieved hemostasis [34]. Whether a high FFP:RBC ratio or early therapy of coagulopathy is beneficial for survival, as shown in some studies, is still unclear based on currently available research $[41,44]$. In light of the very heterogenous bleeding situations, a rigid ratio-driven transfusion protocol is unlikely to accurately address hemostatic requirements in a hemorrhaging patient [45]. Instead, there is growing evidence that viscoelastic coagulation monitoring (with TEG or ROTEM) and goal-directed bleeding management is superior to fixed-ratio transfusion in severe bleeding - in particular in cardiovascular surgery [46-48] and trauma [49-53]. For instance, in a trauma RCT performed in the US, TEG-guided blood transfusion resulted in a significant reduction in plasma transfusion, platelet transfusion and 28 -day mortality (19.6 vs. $36.4 \%$; $p=$
0.0489 [49]. Observational data in patients with postpartum hemorrhage show that the introduction of a ROTEMguided algorithm for treatment of coagulopathy in major obstetric hemorrhage resulted in a significant reduction in the number of units $(p<0.0001)$ and total volume $(p=$ 0.0007 ) of blood products transfused, with a reduction in transfusion-associated circulatory overload (TACO) $(p=$ 0.002) [54].

Generally, the use of plasma-derived and recombinant coagulation factor preparations such as PCC, fibrinogen concentrate and other coagulation factors in massive transfusion has not yet been comprehensively investigated $[55,56]$. However, some data seem to demonstrate an advantage for a clotting factor-based therapy in massive bleeding $[49,50]$. Even though plasma contains all clotting factors, transfusion of FFP in bleeding patients does not achieve sustained correction of coagulation [57]. The most prominent reason for this is that the fibrinogen level is diluted rather than increased when bleeding is treated with plasma as the fibrinogen content of plasma is relatively low compared with cryoprecipitate [14]. As fibrinogen is often the first coagulation factor that declines to critical levels in massive bleeding, FFP and/or thawed plasma are not the best choices for fibrinogen replacement. Although 1 unit of FFP, 1 unit of cryoprecipitate, and one dose of concentrated fibrinogen may all contain a similar amount of fibrinogen, a more concentrated formulation is better able to increase the fibrinogen level to clinical significance. Specifically, 1 unit of plasma adds volume to the circulation but dilutes the concentration of available fibrinogen, thereby decreasing its clinical effect. In 2019, Morrison et al. [58] demonstrated that fibrinogen concentrate may be given instead of FFP to treat coagulopathy during complex vascular surgery and that its use results in reduced allogeneic blood component transfusion.

The RETIC trial published in 2017 also focused on treatment of coagulopathy in major trauma [50]: the authors aimed to compare the efficacy of FFP or clotting factors in reversing trauma-induced coagulopathy, as well as the arising transfusion requirements and development of multiple organ failure in a single-center, parallelgroup, open-label, randomized design. The study was terminated early due to institutional review board-mandated, predefined stopping rules. Specifically, treatment failure in the FFP group reached $>50 \%$ (52 vs. $4 \%$ ) with the need for a cross-over. Patients in the plasma group had a higher rate of diffuse and massive bleeding than patients of the clotting factor group. Plasma transfusion was $100 \%$ in the FFP group and 4\% in the ROTEM-guided coagulation factor concentrate group. The odds for receiving massive transfusion were three-fold higher with plasma. In conclusion, the targeted administration of coagulation factor concentrates was more effective than the 
usual transfusion of fresh plasma in patients with traumainduced coagulopathy.

Currently, there is no evidence that therapies such as therapeutic plasma or cryoprecipitate are safer or more effective for acquired bleeding than other therapies. However, studies addressing such questions are difficult to implement and hardly feasible. Goldstein et al. [59] compared the efficacy and safety of 4F-PCC with that of plasma in 181 VKA-treated patients needing urgent surgical or invasive procedures in a multicenter, open-label, phase $3 \mathrm{~b}$ randomized trial. The primary endpoint was effective hemostasis, which was achieved in 78 (90\%) patients in the 4F-PCC group compared with $61(75 \%)$ patients in the plasma group, demonstrating both non-inferiority and superiority of 4F-PCC over plasma (difference 14.3\%, 95\% CI $2.8-$ 25.8). Rapid INR reduction was achieved in 48 (55\%) patients in the 4F-PCC group compared with 8 (10\%) patients in the plasma group, demonstrating both non-inferiority and superiority of 4F-PCC over plasma (difference $45.3 \%$, 95\% CI 31.9-56.4). The safety profile was similar, but 4FPCC was associated with fewer fluid overload events.

Despite the lack of evidence, the use of FFP remains an established part of the protocols for massive transfusions in most (trauma) centers $[16,60]$, presumably because there is also a lack of evidence demonstrating the superiority of alternative treatment strategies.

Although FFP transfusion is used to treat bleeding patients, there is little robust scientific evidence that transfusion of plasma is effective in a hemostatic treatment; a critical examination of the methodology and conclusions of widely cited RCTs necessitates cautious interpretation of their results. Compared to other therapeutic interventions, no beneficial effects of plasma transfusion in the treatment of massive bleeding have been demonstrated by RCTs, and especially in the most severely injured patients, posttraumatic coagulopathy remains a frequent and life-threatening syndrome [61]. As every bleeding patient is unique, the different needs that occur in massive hemorrhage may not be best addressed simply by transfusing a fixed ratio of blood components. In light of the heterogeneous nature of conditions and their rapid evolvement over time, factor concentrate therapy, directed at specific phases of coagulation identified by alternative laboratory assays, offers the advantage of smaller volumes of resuscitative fluids and, hence, may yield better outcomes. The outcome benefit of factor concentrates, however, needs to be demonstrated in well-powered prospective trials, which is complicated by the legacy of its use, by the difficulty of ethically accurate study planning, and institutional inconsistency of therapeutic procedures. Data from the European multicenter iTACTIC study on coagulation management in traumatic major hemorrhage are awaited soon and may provide further insights [62].

\section{Other Indications}

There are few prospective trials of high quality concerning other potential indications for plasma transfusion in non-bleeding patients.

A non-inferiority randomized control trial recruited 81 non-bleeding critically ill patients with an INR of 1.5-3.0 who were about to undergo an invasive procedure, and randomized patients to receive either FFP $12 \mathrm{~mL} / \mathrm{kg}$ or no FFP [63]. Incidence of bleeding did not differ between groups, with a total of one major and 13 minor bleedings ( $p=0.08$ for noninferiority). FFP transfusion had only a marginally beneficial effect on improving coagulation profiles, as although levels of FII, FV and FVII were elevated, thrombin generation was unaffected and anticoagulant factor levels were elevated. Rocha et al. [64] compared three transfusion protocols prior to central venous catheterization in critically ill cirrhosis patients in a randomized controlled approach, including 57 patients. In this relatively small cohort, no difference in bleeding was found; the restrictive strategy significantly reduced blood transfusion and costs in patients with cirrhosis. However, incidence of bleeding after central venous line insertion using real-time ultrasound guidance for patients with coagulopathy is comparatively low, so the study cohort may have been too small to find significant differences [65]. Nevertheless, this underlines that ultrasound guidance should be mandatory for central venous catheter placement in patients at risk for bleeding, which is presumably much more effective in the prevention of bleeding than any preprocedural correction of hemostasis.

A Cochrane review evaluated the risk to benefit ratio of FFP transfusion in cardiovascular surgery for the treatment of bleeding patients or for prophylaxis against bleeding [66]. Fifteen trials were included in the review with a total of 755 participants for analysis. Fourteen of these trials compared prophylactic use of FFP against no FFP. However, the term "prophylactic FFP transfusion" in this setting included FFP transfusion at the time of heparin neutralization and stopping cardiopulmonary bypass (CPB) (7 trials), with $\mathrm{CPB}$ priming ( 4 trials), after anesthesia induction ( 1 trial) and postoperatively (2 trials), which equals anticipating therapeutic FFP transfusion without laboratory proof of coagulopathy. Generally, studies included in this review varied grossly, i.e. 12 trials excluded patients having emergency surgery and 9 excluded patients with coagulopathies. Additionally, risk of bias was either high or unknown, and only 1 included study reported adverse events as an outcome, limiting the scope and quality of evidence. Keeping these limitations in mind, this review found no evidence that the prophylactic use of FFP affected perioperative blood loss in cardiac surgery and there was a significant increase in the number of patients requiring RBC transfusion who were randomized to FFP. 
In patients with intracranial bleeding, the randomized controlled INCH trial included patients with VKA-associated intracranial hemorrhage and INR levels greater or equal to 2 on hospital admission to compare 4-F-PCC (30 $\mathrm{IU} / \mathrm{kg} \mathrm{BW})$ versus FFP $(20 \mathrm{~mL} / \mathrm{kg}$ BW $)$ both combined with intravenous vitamin $\mathrm{K}(10 \mathrm{mg})$. The primary endpoint was the proportion of patients achieving an INR $\leq 1.2$ within $3 \mathrm{~h}$, and the trial was prematurely stopped after enrolment of 54 patients as hematoma expansion rates significantly differed between treatments [67]. Although an effect of PCC on clinical outcomes remains to be shown, these data favor the use of PCC over FFP in intracranial hemorrhage related to VKA.

We found no controlled trials on the efficacy of plasma transfusion in patients with disseminated intravascular coagulation in adults.

\section{Plasma Transfusion Practice}

According to the German Paul-Ehrlich Institute, reported plasma utilization in Germany has declined from $1,190,327$ units in 2010 to 683,303 units in 2018 [68]. There is a similar trend in the United States from 3,882,000 units in 2011 to 2,727,000 in 2015 [69]. Nevertheless, there is wide heterogeneity in factors associated with plasma transfusion, including hospital type and location [70]. Many institutions are compliant with plasma transfusion guidelines based on published references, supported by appropriate testing [71]. Nevertheless, plasma transfusions for indications that lack evidence of efficacy and incidence of transfusion reactions, especially inappropriate prophylactic transfusion of plasma, still prevail, despite more than a decade of reviews and narratives highlighting lack of evidence for this approach [72, 73]. 24-48\% of all plasma transfusions are reported to be prophylactic transfusions [74]. As there is no evidence validating FFP use in these settings, this practice potentially exposes patients to unnecessary transfusion-associated adverse events.

Triulzi et al. [75] retrospectively analyzed a total of 72,167 units of plasma transfused in 10 US hospitals. The median dose of plasma patients received who were treated for coagulopathy was 2 units (interquartile range, 2-4; range 1-72). This equals a median dose of $7.3 \mathrm{~mL} / \mathrm{kg}$ when adjusted to patient body weight. Only $29 \%$ of doses were at least $10 \mathrm{~mL} / \mathrm{kg}$, and only $15.5 \%$ were at least only $15 \mathrm{~mL} / \mathrm{kg}$. Nearly one-fourth (22.5\%) of all plasma transfusions were given to patients with an INR of less than 1.6. This demonstrates both inadequate indication and suboptimal plasma dosing as Chowdary et al. [76] showed that even doses of $10-15 \mathrm{~mL} / \mathrm{kg}$ resulted in only small (i.e., 10-15\%) increments in coagulation factor levels compared to patients who received $30 \mathrm{~mL} / \mathrm{kg}$ who experienced complete correction of factor levels.
Prevailing inadequate dosing and unnecessary plasma usage for indications not substantiated by robust trial data are common. Owing to time constraints or lack of suitable laboratory tests, transfusion of hemostatic products is often inappropriately triggered, blindly to the coagulation status. This presents PBM programs with ample opportunity to reduce plasma transfusions. Sarode et al. [77] were able to reduce inappropriate plasma usage by $60 \%$ after hospital-wide education and prospective monitoring through transfusion services laboratory staff and pathology house staff and faculty. Real-time clinical decision support system for computerized physician order entry of plasma orders also proved effective [78]. Shah et al. [79] were able to decrease monthly plasma utilization by $17.4 \%$ by instituting clinical decision support as an alert upon order entry if the patient's recent INR was 1.7 or less. Further possibilities to reduce plasma requirements is perioperative point-of-care coagulation management with early calculated goal-directed therapy [80]. Görlinger et al. [80] showed that the implementation of point-of-care coagulation management algorithms with fibrinogen concentrate and PCC resulted in a reduction in transfusion requirements for FFPs by about $90 \%$ in different perioperative settings and reduced the incidence of massive transfusion. A significant reduction of plasma transfusion by viscoelastic testing-guided bleeding management has also been confirmed in other RCTs and meta-analyses [48, 52, 53].

\section{Role of FFP in the Treatment of Coagulopathy}

An imbalance in the coagulation system can not immediately be corrected by the use of FFP. The decisive factor for an effective correction is not the total amount of coagulation factors that are substituted, but their concentration [81]. The concentrations of coagulation factors in FFP correspond at best to physiological conditions and are therefore too low to achieve a relevant change in concentration in coagulopathic and bleeding patients. Individual factor concentrations can vary considerably depending on the donor, as shown above and are finally not known. In addition, it should be considered that no specific correction is achievable with plasma transfusion, since all procoagulants but also anticoagulants are included [40].

Only the administration of large volumes of plasma is able to compensate for the lack of fibrinogen, coagulation factors, the prothrombin complex, and protein $\mathrm{C}$, while at the same time unnecessarily substituting factor VIII and von Willebrand factor. According to guidelines, generally, the recommended dose of FFP required to reverse a coagulopathy is $10-15 \mathrm{~mL} / \mathrm{kg}$ but should not exceed 20 $\mathrm{mL}$ per $\mathrm{kg}$ because transfusing higher plasma volumes confers no significant benefit [82]. 
Plasma transfusion is often used to prevent or stop bleeding by correcting abnormal coagulation results; however, most patients with mildly abnormal coagulation test results do not have a significant hemostatic defect and do not require plasma transfusion. In clinical practice, administration of FFP is usually guided by laboratory tests of coagulation, mainly PT, INR, and aPTT, but the predictive value of these tests to predict bleeding is poor [83]. The use of plasma to correct an abnormal INR is predicated on the assumption that a prolonged INR is associated with an increased risk of bleeding and that plasma therapy will correct the INR. It should be mentioned that the effect of plasma transfusion on the INR is transient. For the same volume of transfused plasma, a greater reduction in INR is observed at higher initial INRs; and the effect of plasma transfusion on INR correction decreases as more plasma is transfused [84].

Additionally, it should be noted that some coagulation factors have a short biological half-life (factor V: 12-15 h; factor VII: 3-6 h). The effect of substitution is not maintained over long periods of time, so short transfusion intervals are necessary to achieve and maintain hemostatically effective plasma levels. Patients with acquired coagulopathies often show an increased turnover of coagulation factors and inhibitors due to consumption and/or loss or dilution, and consequently shorten and reduce effectiveness of the plasma [20].

Only a lack of factor $\mathrm{V}$ can solely be compensated by the administration of FFP, as no factor concentrates are currently available for this purpose. In this rare case, situations of bleeding need to be treated with FFP.

\section{Side Effects of FFP and Plasma-Derived Medicines}

Transfusion of blood products can be lifesaving but also comes with risks and the potential for patient harm. It is fortunate that the risk of transmitting infectious diseases through donor testing, quarantining and pathogen reduction strategies is now considerably reduced $[85,86]$, but a measurable risk for transmitting infectious diseases remains to date.

Hemovigilance systems demonstrated in recent years that transfusion-related acute lung injury (TRALI) is a major cause of transfusion-related morbidity and mortality from plasma transfusions [87]. The knowledge of the origin of TRALI led to changes in the guidelines for plasma collection, which have been shown to reduce the risk of TRALI from plasma transfusion [88-90]. Nevertheless, TRALI still has an estimated incidence of $0.02-1.12 \%$ per transfused blood product and can exceed $5-8 \%$ in critically ill adults and children with a mortality rate of up to $43 \%$ in these high-risk patients [91].
Another side effect is TACO, which gains increasing relevance and attention, although it is presumably still grossly underdiagnosed and underreported with a stated incidence of approximately $1 \%$ in the aftermath of plasma transfusions $[92,93]$. TACO cases are responsible for most deaths and major morbidity reported to hemovigilance, with an increasing number reported year on year [94]. Although TACO has already been reported after a single transfusion [95], plasma transfusions in particular are considered a risk factor for TACO, as they are often administered in larger volumes [96]. Patients at special risk for TACO are those with a history of cardiopulmonary diseases, especially congestive heart failure and/or coronary artery disease, as well as pediatric and elderly patients [97]. In a cohort of mixed intensive care unit patients who received transfusions, TACO was diagnosed in 3.6-5.8\% [98]. Especially in cirrhotic patients, volume overload is also an important reason for bleeding and can be aggravated by FFP transfusion in order to better prevent TACO as a potentially avoidable complication; further studies are needed to establish preventive strategies. In this context, the predictive value of laboratory tests in identifying patients at risk for bleeding should be viewed critically $[99,100]$.

Likewise, allergic reactions, which occur in approximately 1-3\% [101], are causative of various pathogenetic mechanisms and are still not fully understood, requiring further research.

Plasma transfusion may furthermore increase the risk of nosocomial infections in parallel with the development of immune modulation referred to as transfusion-related immunomodulation. In emergency and elective general surgery, trauma, and critical care, plasma transfusion was associated with postoperative infectious complications [102-106]. In support, immunomodulatory effects were shown in vitro in co-cultures of plasma products and human cells from monocytic lineage, demonstrating that both the magnitude and direction of inflammatory cell regulation is influenced $[107,108]$. The effects varied depending on type of plasma product and donor-specific characteristics such as age.

Plasma transfusions are considerably safer today due to a variety of approaches and techniques, but a zero risk is still not achievable. Clinicians need to be aware of the potential risks of plasma transfusions as, unfortunately, a large number of plasma transfusions still occur without an evidence-based indication. In the future, research on the indications for FFP transfusions is warranted to minimize inappropriate plasma transfusions and to determine evidence-based guidelines for plasma transfusions.

Adverse events associated with PCC include immediate allergic reactions, heparin-induced thrombocytopenia (HIT, for the preparations containing heparin) and thromboembolic complications.[109] The primary safety 
concern with PCC is their association with thrombogenic events such as stroke, myocardial infarction, pulmonary embolism, disseminated intravascular coagulation and deep vein thrombosis. The incidence of side effects heavily depends on patients' underlying thrombotic risk factors, dosing, and indication for usage. In a review by Leissinger et al. [110], PCC used for reversing warfarin anticoagulation was associated with a low risk of thrombotic adverse events (7/506 cases, $1.4 \%)$. Others find higher rates of thromboembolic events when comparing 4 F-PCC with FFP (2.7 vs. $17.7 \%, p \leq 0.001)$ [111].

\section{Conclusion}

The evidence for the efficacy of FFP is inconsistent and low across all assessed indications and outcomes. Especially the role (if any) of prophylactic plasma transfusion in non-bleeding patients with abnormalities of standard coagulation tests, which have limited value in predicting bleeding, remains unclear. Prophylactic plasma transfusion offends established guidelines, presenting risks of patient harm, while providing no evident clinical benefit. Our review clearly demonstrates that the use of plasma transfusion in perioperative bleeding and coagulopathy is limited by efficacy, volume, preparation, and the risk of adverse transfusion reactions. Plasma transfusions for INR of less than 1.6 and doses of 1-2 units of plasma should be strongly discouraged. In light of potential side effects, FFP should never be used as a simple volume replacement. Special risk groups for side effects of FFP transfusion should preferentially be treated restrictively or with coagulation factor concentrates.

Evidence-based transfusion practice education, embedded in PBM programs, should especially emphasize the nonlinear relationship between coagulation factor levels and the volume of plasma transfused as well as the profound lack of evidence for plasma transfusions. We furthermore recommend the introduction of electronic interventions to target plasma transfusions for indications in which these are clearly not supported by the current evidence. Treatment algorithms, based on point-ofcare testing and rational use of coagulation factor concentrates, reduce transfusion rates of allogeneic blood products, including plasma. Goal-directed strategies, adapted to local institutional circumstances and resources, and guided either by conventional coagulation tests or viscoelastic assays that take underlying coagulopathy into account, allow individualized therapies tailored to each patient's needs and are likely to achieve better outcomes. As clinicians become fully proficient with viscoelastic assays and transfusion algorithm, adherence to evidencebased treatment strategies will rise, and treatment effects may become clearer in RCTs comparing concentration factor-based coagulation management in acute bleeding situations with FFP-based strategies.

\section{Acknowledgement}

No further contributions to declare.

\section{Conflict of Interest Statement}

E.H.A. received a research grant of the German Research Foundation (AD 592/1-1). D.F. has no conflicts of interest to declare.

\section{Funding Sources}

No external funding declared.

\section{Author Contributions}

Adam E.H. and Fischer D. contributed equally to the interpretation of the literature, editing, and writing of the manuscript.

\section{References}

1 Plasma for Therapeutic Use. 4 Plasma for Therapeutic Use. Transfus Med Hemother. 2009;36(6):388-97.

2 Watson JJ, Pati S, Schreiber MA. Plasma Transfusion: History, Current Realities, and Novel Improvements. Shock. 2016 Nov;46(5): 468-79.

3 Shamsudin S, Yousuf R, Tang YL, Ding CH, Leong CF. Evaluation of coagulation factor activity and sterility of thawed fresh frozen plasma during storage up to 5 days at $4{ }^{\circ} \mathrm{C}$. Malays J Pathol. 2020 Apr;42(1):59-64.
4 Neisser-Svae A, Trawnicek L, Heger A, Mehta T, Triulzi D. Five-day stability of thawed plasma: solvent/detergent-treated plasma comparable with fresh-frozen plasma and plasma frozen within 24 hours. Transfusion. 2016 Feb;56(2):404-9.

5 von Heymann C, Keller MK, Spies C, Schuster M, Meinck K, Sander M, et al. Activity of clotting factors in fresh-frozen plasma during storage at 4 degrees $\mathrm{C}$ over 6 days. Transfusion. 2009 May;49(5):913-20.

6 Runkel S, Haubelt H, Hitzler W, Hellstern P. The quality of plasma collected by automated apheresis and of recovered plasma from leukodepleted whole blood. Transfusion. 2005 Mar;45(3):427-32.
7 Bux J, Dickhörner D, Scheel E. Quality of freeze-dried (lyophilized) quarantined singledonor plasma. Transfusion. $2013 \mathrm{Dec}$;53(12): 3203-9.

8 Garrigue D, Godier A, Glacet A, Labreuche J, Kipnis E, Paris C, et al. French lyophilized plasma versus fresh frozen plasma for the initial management of trauma-induced coagulopathy: a randomized open-label trial. J Thromb Haemost. 2018 Mar;16(3):481-9.

9 Thevis M, Krug O, Geyer H, Wenzel F, Bux J, Stahl L, et al. Monitoring drug residues in donor blood/plasma samples using LC-(MS)/ MS—a pilot study. Drug Test Anal. 2013 May; 5(5):380-3 
10 Rock G. A comparison of methods of pathogen inactivation of FFP. Vox Sang. 2011 Feb; 100(2):169-78.

11 Suontaka AM, Blombäck M, Chapman J. Changes in functional activities of plasma fibrinogen after treatment with methylene blue and red light. Transfusion. 2003 May;43(5): 568-75.

12 Theusinger OM, Baulig W, Seifert B, Emmert MY, Spahn DR, Asmis LM. Relative concentrations of haemostatic factors and cytokines in solvent/detergent-treated and fresh-frozen plasma. Br J Anaesth. 2011 Apr;106(4):50511.

13 Haubelt $\mathrm{H}$, Blome $\mathrm{M}$, Kiessling $\mathrm{AH}$, Isgro $\mathrm{F}$, Bach J, Saggau W, et al. Effects of solvent/detergent-treated plasma and fresh-frozen plasma on haemostasis and fibrinolysis in complex coagulopathy following open-heart surgery. Vox Sang. 2002 Jan;82(1):9-14.

14 Tobin JM, Tanaka KA, Smith CE. Factor concentrates in trauma. Curr Opin Anaesthesiol. 2015 Apr;28(2):217-26.

15 National Blood Authority. Australia. Patient blood management guidelines. Canberra: $\mathrm{Na}$ tional Blood Authority; 2012. Available from: www.nba.gov.au/guidelines/review.html

16 Roback JD, Caldwell S, Carson J, Davenport R, Drew MJ, Eder A, et al.; American Association for the Study of Liver; American Academy of Pediatrics; United States Army; American Society of Anesthesiology; American Society of Hematology. Evidence-based practice guidelines for plasma transfusion. Transfusion. 2010 Jun;50(6):1227-39.

17 Green L, Bolton-Maggs P, Beattie C, Cardigan R, Kallis Y, Stanworth SJ, et al. British Society of Haematology Guidelines on the spectrum of fresh frozen plasma and cryoprecipitate products: their handling and use in various patient groups in the absence of major bleeding. Br J Haematol. 2018 Apr;181(1):54-67.

18 Curry NS, Davenport R, Pavord S, Mallett SV, Kitchen D, Klein AA, et al. The use of viscoelastic haemostatic assays in the management of major bleeding: A British Society for Haematology Guideline. Br J Haematol. 2018 Sep; 182(6):789-806.

19 Kujovich JL. Hemostatic defects in end stage liver disease. Crit Care Clin. 2005 Jul;21(3): 563-87.

20 Executive Committee of the German Medical Association on the Recommendation of the Scientific Advisory Board. Cross-Sectional Guidelines for Therapy with Blood Components and Plasma Derivatives: Chapter $5 \mathrm{Hu}-$ man Albumin - Revised. Transfus Med Hemother. 2016;43(3):223-32

21 Kozek-Langenecker SA, Ahmed AB, Afshari A, Albaladejo P, Aldecoa C, Barauskas G, et al. Management of severe perioperative bleeding: guidelines from the European Society of Anaesthesiology: First update 2016. Eur J Anaesthesiol. 2017 Jun;34(6):332-95.

22 Klein AA, Arnold P, Bingham RM, Brohi K, Clark R, Collis R, et al. AAGBI guidelines: the use of blood components and their alternatives 2016. Anaesthesia. 2016 Jul;71(7):82942.

23 Collins P, Abdul-Kadir R, Thachil J; Subcommittees on Women's Health Issues in Thrombosis and Haemostasis and on Disseminated
Intravascular Coagulation. Management of coagulopathy associated with postpartum hemorrhage: guidance from the SSC of the ISTH. J Thromb Haemost. 2016 Jan;14(1): 205-10.

24 Pagano D, Milojevic M, Meesters MI, Benedetto U, Bolliger D, von Heymann C, et al. 2017 EACTS/EACTA Guidelines on patient blood management for adult cardiac surgery. Eur J Cardiothorac Surg. 2018;53(1):79-111.

25 Raphael J, Mazer CD, Subramani S, Schroeder A, Abdalla M, Ferreira R, et al. Society of Cardiovascular Anesthesiologists Clinical Practice Improvement Advisory for Management of Perioperative Bleeding and Hemostasis in Cardiac Surgery Patients. Anesth Analg. 2019 Nov;129(5):1209-21.

26 Vlaar AP, Oczkowski S, de Bruin S, Wijnberge M, Antonelli M, Aubron C, et al. Transfusion strategies in non-bleeding critically ill adults: a clinical practice guideline from the European Society of Intensive Care Medicine. Intensive Care Med. 2020 Apr;46(4):673-96.

27 Levy JH, Grottke O, Fries D, Kozek-Langenecker S. Therapeutic Plasma Transfusion in Bleeding Patients: A Systematic Review. Anesth Analg. 2017 Apr;124(4):1268-76.

28 Brohi K, Cohen MJ, Davenport RA. Acute coagulopathy of trauma: mechanism, identification and effect. Curr Opin Crit Care. 2007 Dec;13(6):680-5.

29 Maegele M, Lefering R, Paffrath T, Tjardes T, Simanski C, Bouillon B; Working Group on Polytrauma of the German Society of Trauma Surgery (DGU). Red-blood-cell to plasma ratios transfused during massive transfusion are associated with mortality in severe multiple injury: a retrospective analysis from the Trauma Registry of the Deutsche Gesellschaft für Unfallchirurgie. Vox Sang. 2008 Aug;95(2): 112-9.

30 Bhangu A, Nepogodiev D, Doughty H, Bowley DM. Meta-analysis of plasma to red blood cell ratios and mortality in massive blood transfusions for trauma. Injury. $2013 \mathrm{Dec}$; 44(12):1693-9.

31 Holcomb JB, del Junco DJ, Fox EE, Wade CE, Cohen MJ, Schreiber MA, et al.; PROMMTT Study Group. The prospective, observational, multicenter, major trauma transfusion (PROMMTT) study: comparative effectiveness of a time-varying treatment with competing risks. JAMA Surg. 2013 Feb;148(2): 127-36.

32 Holcomb JB, Zarzabal LA, Michalek JE, Kozar RA, Spinella PC, Perkins JG, et al.; Trauma Outcomes Group. Increased platelet:RBC ratios are associated with improved survival after massive transfusion. J Trauma. 2011 Aug; 71(2 Suppl 3):S318-28.

33 Scalea TM, Bochicchio KM, Lumpkins K, Hess JR, Dutton R, Pyle A, et al. Early aggressive use of fresh frozen plasma does not improve outcome in critically injured trauma patients. Ann Surg. 2008 Oct;248(4):578-84.

34 Holcomb JB, Tilley BC, Baraniuk S, Fox EE, Wade CE, Podbielski JM, et al.; PROPPR Study Group. Transfusion of plasma, platelets, and red blood cells in a 1:1:1 vs a 1:1:2 ratio and mortality in patients with severe trauma: the PROPPR randomized clinical trial. JAMA. 2015 Feb;313(5):471-82.
35 Sperry JL, Guyette FX, Brown JB, Yazer MH, Triulzi DJ, Early-Young BJ, et al.; PAMPer Study Group. Prehospital Plasma during Air Medical Transport in Trauma Patients at Risk for Hemorrhagic Shock. N Engl J Med. 2018 Jul;379(4):315-26.

36 Moore HB, Moore EE, Chapman MP, McVaney K, Bryskiewicz G, Blechar R, et al. Plasma-first resuscitation to treat haemorrhagic shock during emergency ground transportation in an urban area: a randomised trial. Lancet. 2018 Jul;392(10144):283-91.

37 Fenger-Eriksen C, Fries D, David JS, Bouzat P, Lance MD, Grottke O, et al. Pre-hospital plasma transfusion: a valuable coagulation support or an expensive fluid therapy? Crit Care. 2019 Jul;23(1):238.

38 Jost D, Lemoine S, Lemoine F, Lanoe V, Maurin O, Derkenne C, et al.; PREHO-PLYO Study Group. French lyophilized plasma versus normal saline for post-traumatic coagulopathy prevention and correction: PREHO-PLYO protocol for a multicenter randomized controlled clinical trial. Trials. 2020 Jan;21(1):106.

39 Stanworth SJ, Brunskill SJ, Hyde CJ, McClelland DB, Murphy MF. Is fresh frozen plasma clinically effective? A systematic review of randomized controlled trials. Br J Haematol. 2004 Jul;126(1):139-52.

40 Yang L, Stanworth S, Hopewell S, Doree C, Murphy M. Is fresh-frozen plasma clinically effective? An update of a systematic review of randomized controlled trials [quiz]. Transfusion. 2012 Aug;52(8):1673-86.

41 Murad MH, Stubbs JR, Gandhi MJ, Wang AT, Paul A, Erwin PJ, et al. The effect of plasma transfusion on morbidity and mortality: a systematic review and meta-analysis. Transfusion. 2010 Jun;50(6):1370-83.

42 McQuilten ZK, Crighton G, Brunskill S, Morison JK, Richter TH, Waters N, et al. Optimal Dose, Timing and Ratio of Blood Products in Massive Transfusion: Results from a Systematic Review. Transfus Med Rev. 2018 Jan; 32(1):6-15.

43 da Luz LT, Shah PS, Strauss R, Mohammed AA, D'Empaire PP, Tien $\mathrm{H}$, et al. Does the evidence support the importance of high transfusion ratios of plasma and platelets to red blood cells in improving outcomes in severely injured patients: a systematic review and meta-analyses. Transfusion. 2019 Nov; 59(11):3337-49.

44 Snyder CW, Weinberg JA, McGwin G Jr, Melton SM, George RL, Reiff DA, et al. The relationship of blood product ratio to mortality: survival benefit or survival bias? J Trauma. 2009 Feb;66(2):358-62; discussion 362-4.

45 Goobie SM, Shander A. One Size Does Not Fit All in Treating Massive Hemorrhage. Anesth Analg. 2020 Aug;131(2):480-2.

46 Weber CF, Görlinger K, Meininger D, Herrmann E, Bingold T, Moritz A, et al. Point-ofcare testing: a prospective, randomized clinical trial of efficacy in coagulopathic cardiac surgery patients. Anesthesiology. 2012 Sep;117(3):53147.

47 Haensig M, Kempfert J, Kempfert PM, Girdauskas E, Borger MA, Lehmann S. Thrombelastometry guided blood-component therapy after cardiac surgery: a randomized study. BMC Anesthesiol. 2019 Nov;19(1):201. 
48 Deppe AC, Weber C, Zimmermann J, Kuhn EW, Slottosch I, Liakopoulos OJ, et al. Pointof-care thromboelastography/thromboelastometry-based coagulation management in cardiac surgery: a meta-analysis of 8332 patients. J Surg Res. 2016 Jun;203(2):424-33.

49 Gonzalez E, Moore EE, Moore HB, Chapman MP, Chin TL, Ghasabyan A, et al. Goal-directed Hemostatic Resuscitation of Traumainduced Coagulopathy: A Pragmatic Randomized Clinical Trial Comparing a Viscoelastic Assay to Conventional Coagulation Assays. Ann Surg. 2016 Jun;263(6):1051-9.

50 Innerhofer P, Fries D, Mittermayr M, Innerhofer N, von Langen D, Hell T, et al. Reversal of trauma-induced coagulopathy using firstline coagulation factor concentrates or fresh frozen plasma (RETIC): a single-centre, parallel-group, open-label, randomised trial. Lancet Haematol. 2017 Jun;4(6):e258-71.

51 Stein P, Kaserer A, Sprengel K, Wanner GA, Seifert B, Theusinger OM, et al. Change of transfusion and treatment paradigm in major trauma patients. Anaesthesia. 2017 Nov; 72(11):1317-26.

52 Wikkelsø A, Wetterslev J, Møller AM, Afshari A. Thromboelastography (TEG) or thromboelastometry (ROTEM) to monitor haemostatic treatment versus usual care in adults or children with bleeding. Cochrane Database Syst Rev. 2016 Aug;(8):CD007871.

53 Santos AS, Oliveira AJ, Barbosa MC, Nogueira JL. Viscoelastic haemostatic assays in the perioperative period of surgical procedures: systematic review and meta-analysis. J Clin Anesth. 2020 Apr;64:109809.

54 McNamara H, Kenyon C, Smith R, Mallaiah S, Barclay P. Four years' experience of a ROTEM $^{\circledR}$-guided algorithm for treatment of coagulopathy in obstetric haemorrhage. Anaesthesia. 2019 Aug;74(8):984-91.

55 Johansson PI, Stensballe J, Oliveri R, Wade CE, Ostrowski SR, Holcomb JB. How I treat patients with massive hemorrhage. Blood. 2014 Nov;124(20):3052-8.

56 Nienaber U, Innerhofer P, Westermann I, Schöchl H, Attal R, Breitkopf R, et al. The impact of fresh frozen plasma vs coagulation factor concentrates on morbidity and mortality in trauma-associated haemorrhage and massive transfusion. Injury. 2011 Jul;42(7):697-701.

57 Khan S, Davenport R, Raza I, Glasgow S, De'Ath HD, Johansson PI, et al. Damage control resuscitation using blood component therapy in standard doses has a limited effect on coagulopathy during trauma hemorrhage. Intensive Care Med. 2015 Feb;41(2):239-47.

58 Morrison GA, Koch J, Royds M, McGee D, Chalmers RT, Anderson J, et al. Fibrinogen concentrate vs. fresh frozen plasma for the management of coagulopathy during thoraco-abdominal aortic aneurysm surgery: a pilot randomised controlled trial. Anaesthesia. 2019 Feb;74(2):180-9.

59 Goldstein JN, Refaai MA, Milling TJ Jr, Lewis B, Goldberg-Alberts R, Hug BA, et al. Fourfactor prothrombin complex concentrate versus plasma for rapid vitamin $\mathrm{K}$ antagonist reversal in patients needing urgent surgical or invasive interventions: a phase $3 \mathrm{~b}$, open-label, non-inferiority, randomised trial. Lancet. 2015 May;385(9982):2077-87.
60 Spahn DR, Bouillon B, Cerny V, Duranteau J, Filipescu D, Hunt BJ, et al. The European guideline on management of major bleeding and coagulopathy following trauma: fifth edition. Crit Care. 2019;23(1):98.

61 Fröhlich M, Mutschler M, Caspers M, Nienaber U, Jäcker V, Driessen A, et al.; TraumaRegister DGU. Trauma-induced coagulopathy upon emergency room arrival: still a significant problem despite increased awareness and management? Eur J Trauma Emerg Surg. 2019 Feb;45(1):115-24.

62 Baksaas-Aasen K, Gall L, Eaglestone S, Rourke C, Juffermans NP, Goslings JC, et al. iTACTIC - implementing Treatment Algorithms for the Correction of Trauma-Induced Coagulopathy: study protocol for a multicentre, randomised controlled trial. Trials. 2017 Oct; 18(1):486.

63 Müller MC, Arbous MS, Spoelstra-de Man AM, Vink R, Karakus A, Straat M, et al. Transfusion of fresh-frozen plasma in critically ill patients with a coagulopathy before invasive procedures: a randomized clinical trial (CME). Transfusion. 2015 Jan;55(1):26-35.

64 Rocha LL, Neto AS, Pessoa CM, Almeida MD, Juffermans NP, Crochemore T, et al. Comparison of three transfusion protocols prior to central venous catheterization in patients with cirrhosis: A randomized controlled trial. J Thromb Haemost. 2020 Mar;18(3):560-70.

65 van de Weerdt EK, Biemond BJ, Baake B, Vermin B, Binnekade JM, van Lienden KP, et al. Central venous catheter placement in coagulopathic patients: risk factors and incidence of bleeding complications. Transfusion. 2017 Oct;57(10):2512-25.

66 Desborough M, Sandu R, Brunskill SJ, Doree C, Trivella M, Montedori A, et al. Fresh frozen plasma for cardiovascular surgery. Cochrane Database Syst Rev. 2015 Jul;(7):CD007614.

67 Steiner T, Poli S, Griebe M, Hüsing J, Hajda J, Freiberger A, et al. Fresh frozen plasma versus prothrombin complex concentrate in patients with intracranial haemorrhage related to vitamin K antagonists (INCH): a randomised trial. Lancet Neurol. 2016 May;15(6):566-73.

68 Paul-Ehrlich-Institut. Reports pursuant to $\$$ 21 TFG. Langen: Paul-Ehrlich-Institut.

69 Ellingson KD, Sapiano MR, Haass KA, Savinkina AA, Baker ML, Chung KW, et al. Continued decline in blood collection and transfusion in the United States-2015. Transfusion. 2017 Jun;57 Suppl 2:1588-98.

70 Goel R, Patel EU, White JL, Chappidi MR, Ness PM, Cushing MM, et al. Factors associated with red blood cell, platelet, and plasma transfusions among inpatient hospitalizations: a nationally representative study in the United States. Transfusion. 2019 Feb;59(2): 500-7.

71 Alcorn K, Ramsey G, Souers R, Lehman CM. Appropriateness of Plasma Transfusion: A College of American Pathologists Q-Probes Study of Guidelines, Waste, and Serious Adverse Events. Arch Pathol Lab Med. 2017 Mar; 141(3):396-401.

72 Zakeri N, Tsochatzis EA. Bleeding Risk with Invasive Procedures in Patients with Cirrhosis and Coagulopathy. Curr Gastroenterol Rep. 2017 Sep;19(9):45.
73 Desborough MJ, Hockley B, Sekhar M, Burroughs AK, Stanworth SJ, Jairath V, et al. Patterns of blood component use in cirrhosis: a nationwide study. Liver Int. 2016;36(4):5229. https://doi.org/10.1111/liv.12999.

74 Tinmouth A. Assessing the Rationale and Effectiveness of Frozen Plasma Transfusions: An Evidence-based Review. Hematol Oncol Clin North Am. 2016 Jun;30(3):561-72.

75 Triulzi D, Gottschall J, Murphy E, Wu Y, Ness P, Kor D, et al.; NHLBI Recipient Epidemiology and Donor Evaluation Study-III (REDSIII). A multicenter study of plasma use in the United States. Transfusion. 2015 Jun;55(6): 1313-9.

76 Chowdary P, Saayman AG, Paulus U, Findlay GP, Collins PW. Efficacy of standard dose and $30 \mathrm{ml} / \mathrm{kg}$ fresh frozen plasma in correcting laboratory parameters of haemostasis in critically ill patients. Br J Haematol. 2004 Apr; 125(1):69-73

77 Sarode R, Refaai MA, Matevosyan K, Burner JD, Hampton S, Rutherford C. Prospective monitoring of plasma and platelet transfusions in a large teaching hospital results in significant cost reduction. Transfusion. 2010 Feb;50(2):487-92.

78 Yazer MH, Triulzi DJ, Reddy V, Waters JH. Effectiveness of a real-time clinical decision support system for computerized physician order entry of plasma orders. Transfusion. 2013 Dec;53(12):3120-7.

79 Shah N, Baker SA, Spain D, Shieh L, Shepard J, Hadhazy E, et al. Real-Time Clinical Decision Support Decreases Inappropriate Plasma Transfusion. Am J Clin Pathol. 2017 Aug; 148(2):154-60.

80 Görlinger K, Fries D, Dirkmann D, Weber CF, Hanke AA, Schöchl H. Reduction of Fresh Frozen Plasma Requirements by Perioperative Point-of-Care Coagulation Management with Early Calculated Goal-Directed Therapy. Transfus Med Hemother. 2012 Apr; 39(2):104-13.

81 Holland LL, Foster TM, Marlar RA, Brooks JP. Fresh frozen plasma is ineffective for correcting minimally elevated international normalized ratios. Transfusion. 2005 Jul;45(7): 1234-5.

82 Yazer MH. The how's and why's of evidence based plasma therapy. Korean J Hematol. 2010 Sep;45(3):152-7.

83 Segal JB, Dzik WH; Transfusion Medicine/ Hemostasis Clinical Trials Network. Paucity of studies to support that abnormal coagulation test results predict bleeding in the setting of invasive procedures: an evidence-based review. Transfusion. 2005 Sep;45(9):1413-25.

84 Bryan AW Jr, Staley EM, Kennell T Jr, Feldman AZ, Williams LA 3rd, Pham HP. Plasma Transfusion Demystified: A Review of the Key Factors Influencing the Response to Plasma Transfusion. Lab Med. 2017 May;48(2): 108-12.

85 Zou S, Dorsey KA, Notari EP, Foster GA Krysztof DE, Musavi F, et al. Prevalence, incidence, and residual risk of human immunodeficiency virus and hepatitis $C$ virus infections among United States blood donors since the introduction of nucleic acid testing. Transfusion. 2010 Jul;50(7):1495-504. 
86 MacLennan S, Williamson LM. Risks of fresh frozen plasma and platelets. J Trauma. 2006 Jun;60(6 Suppl):S46-50.

87 Mayr WR. Haemovigilance: are there significant differences among plasma products? Transfus Apheresis Sci. 2010 Dec;43(3):4079.

88 Müller MC, van Stein D, Binnekade JM, van Rhenen DJ, Vlaar AP. Low-risk transfusionrelated acute lung injury donor strategies and the impact on the onset of transfusion-related acute lung injury: a meta-analysis. Transfusion. 2015 Jan;55(1):164-75.

89 Funk MB, Guenay S, Lohmann A, Henseler O, Heiden M, Hanschmann KM, et al. Benefit of transfusion-related acute lung injury riskminimization measures-german haemovigilance data (2006-2010). Vox Sang. 2012 May; 102(4):317-23.

90 Lin Y, Saw CL, Hannach B, Goldman M. Transfusion-related acute lung injury prevention measures and their impact at Canadian Blood Services. Transfusion. 2012 Mar;52(3): 567-74.

91 McVey MJ, Kapur R, Cserti-Gazdewich C, Semple JW, Karkouti K, Kuebler WM. Transfusion-related Acute Lung Injury in the Perioperative Patient. Anesthesiology. 2019 Sep; 131(3):693-715.

92 Raval JS, Mazepa MA, Russell SL, Immel CC, Whinna HC, Park YA. Passive reporting greatly underestimates the rate of transfusion-associated circulatory overload after platelet transfusion. Vox Sang. 2015 May; 108(4):387-92.

93 Li G, Rachmale S, Kojicic M, Shahjehan K, Malinchoc M, Kor DJ, et al. Incidence and transfusion risk factors for transfusion-associated circulatory overload among medical intensive care unit patients. Transfusion. 2011 Feb;51(2):338-43.

94 Bolton-Maggs PH, Cohen H. Serious Hazards of Transfusion (SHOT) haemovigilance and progress is improving transfusion safety. $\mathrm{Br}$ Haematol. 2013 Nov;163(3):303-14.
95 Rana R, Fernández-Pérez ER, Khan SA, Rana S, Winters JL, Lesnick TG, et al. Transfusion-related acute lung injury and pulmonary edema in critically ill patients: a retrospective study. Transfusion. 2006 Sep;46(9): 1478-83.

96 Narick C, Triulzi DJ, Yazer MH. Transfusion-associated circulatory overload after plasma transfusion. Transfusion. 2012 Jan; 52(1):160-5.

97 Thalji L, Thum D, Weister TJ, Weber WV, Stubbs JR, Kor DJ, et al. Incidence and Epidemiology of Perioperative Transfusion-Related Pulmonary Complications in Pediatric Noncardiac Surgical Patients: A Single-Center, 5-Year Experience. Anesth Analg. 2018 Nov;127(5):1180-8.

98 Bosboom JJ, Klanderman RB, Zijp M, Hollmann MW, Veelo DP, Binnekade JM, et al. Incidence, risk factors, and outcome of transfusion-associated circulatory overload in a mixed intensive care unit population: a nested case-control study. Transfusion. 2018 Feb;58(2):498-506.

99 Dötsch TM, Dirkmann D, Bezinover D, Hartmann M, Treckmann JW, Paul A, et al. Assessment of standard laboratory tests and rotational thromboelastometry for the prediction of postoperative bleeding in liver transplantation. Br J Anaesth. 2017 Sep; 119(3):402-10.

100 O’Leary JG, Greenberg CS, Patton HM, Caldwell SH. AGA Clinical Practice Update: coagulation in Cirrhosis. Gastroenterology. 2019 Jul;157(1):34-43.e1.

101 Gilstad CW. Anaphylactic transfusion reactions. Curr Opin Hematol. 2003 Nov;10(6): 419-23.

102 Ming Y, Liu J, Zhang F, Chen C, Zhou L, Du L, et al. Transfusion of Red Blood Cells, Fresh Frozen Plasma, or Platelets Is Associated With Mortality and Infection After Cardiac Surgery in a Dose-Dependent Manner. Anesth Analg. 2020 Feb;130(2):488-97.

103 Subramanian A, Berbari EF, Brown MJ, Allen MS, Alsara A, Kor DJ. Plasma transfusion is associated with postoperative infectious complications following esophageal resection surgery: a retrospective cohort study. J Cardiothorac Vasc Anesth. 2012 Aug;26(4):569-74.
104 Inaba K, Branco BC, Rhee P, Holcomb JB, Blackbourne LH, Shulman I, et al. Impact of ABO-identical vs ABO-compatible nonidentical plasma transfusion in trauma patients. Arch Surg. 2010 Sep;145(9):899-906.

105 Inaba K, Branco BC, Rhee P, Blackbourne LH, Holcomb JB, Teixeira PG, et al. Impact of plasma transfusion in trauma patients who do not require massive transfusion. J Am Coll Surg. 2010 Jun;210(6):957-65.

106 Sarani B, Dunkman WJ, Dean L, Sonnad S, Rohrbach JI, Gracias VH. Transfusion of fresh frozen plasma in critically ill surgical patients is associated with an increased risk of infection. Crit Care Med. 2008 Apr;36(4): 1114-8

107 Shah S, Coppolino K, Menocha S, Beceiro S, Nateri J, Spinella PC, et al. Immunomodulatory effects of plasma products on monocyte function in vitro. J Trauma Acute Care Surg. 2018;84(6S Suppl 1):S47-S53. https://doi. org/10.1097/TA.0000000000001820.

108 Patlán M, Sánchez-Muñoz F, AmezcuaGuerra LM, Granados A, Páez A, Massó F, et al. Effect of fresh frozen plasma on the in vitro activation of U937 monocytes: a potential role for the age of blood donors and their underlying cytokine profile. Biol Res. 2017 Dec;50(1):42.

109 Ansell J, Hirsh J, Hylek E, Jacobson A, Crowther M, Palareti G. Pharmacology and management of the vitamin $\mathrm{K}$ antagonists: American College of Chest Physicians Evidence-Based Clinical Practice Guidelines (8th Edition). Chest. 2008;133(6 Suppl): 160S-98S.

110 Leissinger CA, Blatt PM, Hoots WK, Ewenstein B. Role of prothrombin complex concentrates in reversing warfarin anticoagulation: a review of the literature. Am J Hematol. 2008 Feb;83(2):137-43.

111 Maguire M, Fuh L, Goldstein JN, Marshall AL, Levine M, Howell ML, et al. Thromboembolic Risk of 4-Factor Prothrombin Complex Concentrate versus Fresh Frozen Plasma for Urgent Warfarin Reversal in the Emergency Department. West J Emerg Med. 2019 Jul;20(4):619-25. 